\title{
Ejercicio físico en adultos mayores incorporados a la Casa de Abuelos.
}

Physical exercise in older adults incorporated into the Grandparents' House.

\author{
Reynier Soria Pérez. ${ }^{1}$, Joanna de Armas Mestre. ${ }^{2}$, Dunia Justa Díaz Camellón. ${ }^{3} \&$ \\ Yudisleyvis Santana García. ${ }^{4}$
}

\section{Abstract.}

Introduction: Physical exercise causes physiological, psychological and social benefits. In Cuba, the Geriatric Education Centers and the Grandparent's House were created to promote their social integration and offer them special health education; incorporate them into physical, recreational and creative activities. Objective: to verify the influence of physical exercise in the elderly of the Grandparent's House of Cárdenas municipality in 2019. Method: descriptive study. The universe that coincides with the

\section{Resumen.}

Introducción: El ejercicio físico provoca beneficios fisiológicos, psicológicos y sociales. En Cuba se crearon los Centros de Educación Geriátrica y Casa de Abuelos para favorecer su integración social y ofrecerles educación sanitaria especial; incorporarlos a las actividades físicas, recreativas y creativas. Objetivo: comprobar la influencia del ejercicio físico en los adultos mayores de la Casa de Abuelos del municipio Cárdenas en el año 2019. Método: estudio descriptivo. El universo que coincide con la muestra

1 Hospital Dr. Julio M. Aristegui Villamil. Cárdenas, Cuba, rsoria.mtz@infomed.sld.cu (iD https://orcid.org/0000-0002-3512-878X

2 Hospital Dr. Julio M. Aristegui Villamil. Cárdenas, Cuba, jdearmas.mtz@infomed.sld.cu (iD https://orcid.org/0000-0003-1946-6495

3 Policlínico Universitario José Antonio Echeverría. Cárdenas, Cuba, dunia.mtz@infomed.sld.cu https://orcid.org/0000-0002-3480-8767

4 Policlínico Universitario José Antonio Echeverría. Cárdenas, Cuba, dunia.mtz@infomed.sld.cu https://orcid.org/0000-0001-9689-1191 
sample was made up of all older adults, with prior informed consent. An interview was designed for data collection. The variables were used: age, gender, personal pathological history, length of stay, history of physical exercises before joining the Grandparent's House and perception of the benefits received. Results: The age group of 60-74 years predominated, and the male gender. Osteomyoarticular diseases prevailed, and the residence time of 1 - 5 years. The largest number of older adults did not perform physical exercises before joining the institution. The most prevalent reported benefit was improvement in interpersonal relationships and ease of movement.

Keywords: Elderly, Physical exercises, permanence, benefits. estuvo constituido por la totalidad de los adultos mayores, previo consentimiento informado. Para la recolección de los datos se diseñó una entrevista. Se utilizaron las variables: edad, género, antecedentes patológicos personales, tiempo de permanencia, antecedentes de ejercicios físicos antes de su incorporación a la Casa de Abuelos y percepción de los beneficios recibidos. Resultados: Predominó el grupo de edad de 60-74 años, y el género masculino. Prevalecieron las enfermedades osteomioarticulares, y el tiempo de permanencia de $1-5$ años. El mayor número de adultos mayores no realizaban ejercicios físicos antes de su incorporación a la institución. El beneficio reportado con más prevalencia fue la mejoría en las relaciones interpersonales y en la facilidad de los movimientos.

Palabras claves: Adulto Mayor, Ejercicios físicos, permanencia, beneficios.

\section{Introducción.}

La población de ancianos mayores de 60 años variará de un 10,4\% a un 21,7\% en el 2050 y los mayores de 80 años aumentarán un 3\%. En los próximos 30 o 40 años, el porcentaje de adultos mayores de 70 años se duplicará mundialmente. (Albizu-Campos Espiñeira, 2019) (Cantillo Bustillo, Rodríguez Pérez, Martínez Cantillo, \& Padilla Martínez, 2019).

La población cubana excede los 11,2 millones de habitantes y presenta una dinámica poblacional característica de un país de transición con demografía avanzada. Se constata un mayor envejecimiento en las provincias occidentales y centrales, lo que se encuentra relacionado con los niveles de fecundidad, mortalidad y las migraciones. Las provincias más envejecidas continúan siendo Ciudad de La Habana y Holguín, mientras que Matanzas ocupa el séptimo lugar. \%. (Oficina Nacional de Estadística e Información, 2019).

En el 2025, Cuba será el país más envejecido de América Latina y en el 2050, uno de los más envejecidos del mundo. (Zavala G, Vidal G, Catro S, Quiroga, \& Klasen P, 2006).

La solución a los problemas de salud de las personas adultas, exige un cambio en la cultura sanitaria, la promoción de salud, su detección temprana y los recursos apropiados para proporcionar rehabilitación comunitaria. En Cuba se crearon los Centros de Educación Geriátrica y las Casas de Abuelo, para favorecer su integración social y ofrecerles 
educación sanitaria especial; Incorporar las actividades físicas, recreativas y creativas. Los beneficios obtenidos reportan mayor percepción de bienestar y adaptación social. (Godoy del LLano, Casanova Moreno, Alvarez Lauzarique, Oliva González, \& Rodríguez Hernández, 2018).

El ejercicio físico provoca beneficios fisiológicos, psicológicos y sociales. Estos se evalúan por mediciones antropométricas, bioquímicas o fisiológicas y por reportes de autoobservación, lo que permite mejorar la calidad de vida del adulto mayor. (Albizu-Campos Espiñeira, 2019).

En Cuba alrededor del 37,2 \% de adultos mayores está incorporado a las Casas de Abuelos, la provincia Matanzas reporta un 31,6 \% y Cárdenas un 26,5 \%. (Oficina Nacional de Estadística e Información, 2019).

Teniendo en cuenta los postulados introductorios del tema y su necesidad de estudio, se plantea como Problema Científico: ¿Qué beneficios perciben los adultos mayores incorporados a la Casa de Abuelos del Municipio Cárdenas durante el año 2019? Se propone como Objetivo General: Comprobar la influencia del ejercicio físico en los adultos mayores incorporados a la Casa de Abuelos del municipio Cárdenas en el año 2019.

\section{Metodologia.}

Se realizó un estudio descriptivo y prospectivo para comprobar la influencia del ejercicio físico en los adultos mayores, incorporados a La casa de Abuelos del Municipio Cárdenas en el año 2019. El universo coincidió con la muestra, constituido por los 49 pacientes matriculados, previo consentimiento informado.

Definiciones de variables según tipos:

- Edad: cuantitativa, continua.

- Género: Variable cualitativa, nominal, dicotómica.

- Antecedentes Patológicos personales, Tiempo de permanencia: cualitativa, ordinal.

- Antecedentes de práctica de ejercicio físico: cualitativa, nominal, dicotómica.

- Beneficios logrados por el ejercicio físico: cualitativa, nominal, politómica.

Consideraciones Éticas.

Se le solicitó el consentimiento cumpliendo lo establecido en los principios de la bioética médica.

Los resultados fueron presentados y expuestos en tablas y comparados con la bibliografía consultada al efecto, llegando a conclusiones.

\section{Resultados.}

Al observar la edad y el género de los integrantes de la Casa de Abuelos (Tabla 1) se muestra un predominio del grupo de 60 - 74 años para un $69,4 \%$, seguido de la edad de 75 
- 89 años lo que representó un 24,5\%. En relación al género, predominó el masculino con 30 pacientes, lo que significó el 61,2\%.

Tabla 1: Distribución de los Adultos Mayores según edad y género. Casa de Abuelos. Municipio Cárdenas. Año 2019.

\begin{tabular}{lcccccc}
\hline \multirow{2}{*}{ Grupos de edades } & \multicolumn{2}{c}{ Masculino } & \multicolumn{2}{c}{ Femenino } & \multicolumn{2}{c}{ Total } \\
\cline { 2 - 7 } & No. & $\%$ & No. & $\%$ & No. & $\%$ \\
\hline $\mathbf{6 0}-\mathbf{7 4}$ años & 25 & 51,0 & 9 & 18,4 & 34 & 69,4 \\
$\mathbf{7 5}-\mathbf{8 9}$ años & 5 & 10,2 & 7 & 14,3 & 12 & 24,5 \\
$\mathbf{9 0}$ y más años & 0 & 0,0 & 3 & 6,1 & 3 & 6,1 \\
Total & 30 & 61,2 & 19 & 38,8 & 49 & 100 \\
\hline
\end{tabular}

Fuente: Elaboración propia

Cuando se evaluaron los antecedentes patológicos personales (Tabla 2) resultó que el 100 $\%$ de los casos refirieron tener alguna enfermedad osteomioarticular seguida de la HTA padecida por el $77,6 \%$.

Tabla 2. Distribución de los Adultos Mayores según Antecedentes Patológicos Personales. Casa de Abuelos. Municipio Cárdenas. Año 2019.

\begin{tabular}{lcc}
\hline Antecedentes Patológicos Personales & No. & $\%$ \\
\hline Enfermedades Osteomioarticulares & 49 & 100 \\
H. T. A. & 38 & 77,6 \\
D. Mellitas & 5 & 10,2 \\
Otras & 3 & 6,1 \\
\hline
\end{tabular}

Fuente: Elaboración propia

Al analizar el tiempo de permanencia en la Casa de Abuelos (Tabla 3) la mayor cantidad de ancianos estuvo entre 1 - 5 años para un 53,1 \%, se observa además una disminución de la misma en la medida que avanza el tiempo, pues durante más de 5 años se extendieron 16 gerontes para un $32,6 \%$.

Tabla 3. Distribución de los Adultos Mayores según Tiempo de Permanencia en la Casa de Abuelos. Municipio Cárdenas. Año 2019.

\begin{tabular}{lcc}
\hline Tiempo de Permanencia & No. & $\%$ \\
Menos de 1 año & 7 & 14,3 \\
$\mathbf{1}-\mathbf{5}$ años & 26 & 53,1 \\
Más de 5 años & 16 & 32,6 \\
Total & 49 & 100 \\
\hline
\end{tabular}

Fuente: Elaboración propia 
En los antecedentes de ejercicio físico antes de su incorporación a la Casa de Abuelos (Tabla 4), se observó que el 75,5\% de los adultos mayores no practicaban ningún tipo de actividad física, solo el $24,5 \%$ lo realizaban.

Tabla 4: Distribución de los Adultos Mayores según Antecedentes de Ejercicio Físico antes de su Incorporación a la Casa de Abuelos. Municipio Cárdenas. Año 2019.

\begin{tabular}{ccc}
\hline Antecedentes de Ejercicio Físico & No. & $\%$ \\
\hline Si & 12 & 24,5 \\
No & 37 & 75,5 \\
Total & 49 & 100 \\
\hline
\end{tabular}

Fuente: Elaboración propia

Interesados por indagar los beneficios que les reportó realizar ejercicios físicos a los adultos mayores en la Casa de Abuelos (Tabla 5) el $100 \%$ de ellos consideró que les mejoró las relaciones interpersonales y la facilidad de movimiento, un $98 \%$ refirió que disminuyeron dolores articulares y el 95,9 \% que la autoestima fue superior. Ninguno de los entrevistados confirmó no tener beneficios tras la realización de la actividad física.

Tabla 5: Distribución de los Adultos Mayores según Percepción propia de los Beneficios del ejercicio físico con la incorporación a la Casa de Abuelos. Municipio Cárdenas. Año 2019.

\begin{tabular}{lcc}
\hline $\begin{array}{l}\text { Percepción por el Adulto Mayor de los Beneficios de } \\
\text { la Incorporación a los Círculos de Abuelos }\end{array}$ & No. & \% \\
\hline Mejora las relaciones interpersonales & 49 & 100 \\
Mejora la facilidad de movimientos & 49 & 100 \\
Mejora los dolores articulares & 48 & 98 \\
Mejora la autoestima & 47 & 95,9 \\
Mejora la circulación & 45 & 91,8 \\
Mejora la fuerza muscular & 43 & 87,8 \\
Mejora la calidad del sueño & 39 & 79,6 \\
Mejora el estado de ánimo & 39 & 79,6 \\
Ningún beneficio & 0 & 0,0 \\
\hline Fuente: Elaboración propia & &
\end{tabular}

Generalmente los adultos mayores que más participan en las Casas de Abuelos están comprendidos entre 60 a 74 años debido a que en edades superiores se dificulta la realización de ejercicios físicos, por los cambios que el envejecimiento produce. Estos resultados coinciden con la literatura consultada. (Piña Moreno, Cordero Ortero, Rodríguez Lemus, Izquiero Izquierdo, \& Vega Pérez, 2019)

En relación al género, en Cuba, (Oficina Nacional de Estadística e Información, 2019) la población adulta es mayor en la mujer que en el hombre, se consideran importantes estos 
datos para realizar trabajo promocional y lograr mayor incorporación de los hombres, siendo una alerta para médicos y enfermeras de la familia, pues resulta de interés tener en cuenta que la incorporación de ejercicios en las Casas de Abuelos tiene como propósito que los gerentes se mantengan funcionales, no coincidiendo con los resultados de este estudio donde la población masculina es mayor.

(Escalante Candeaux, Medina Alvarez, Pila Hernández, \& Gómez Valdés, 2019) encontró prevalencia del género femenino con un 55,8 \%. Resultados similares obtuvo (Ortega Marquez, 2010), con un 66,6 \%. (Hidalgo Feria, 2006) en seis círculos de abuelos de Tacajó obtuvo que el género femenino duplicó al masculino. En otras bibliografías consultadas se encontró que el género femenino, evidencia una tendencia a mantener una mayor esperanza de vida que el masculino, lo que puede atribuirse a la mayor mortalidad masculina por diversos factores. (Godoy del LLano, Casanova Moreno, Alvarez Lauzarique, Oliva González, \& Rodríguez Hernández , 2018) (Montes de Oca García, Bravo, \& Díaz Medina, 2008).

La actividad física es un arma terapéutica inocua y eficaz en la prevención de enfermedades osteomioarticulares. (Montes de Oca García, Bravo, \& Díaz Medina, 2008) En la última década, trabajos controlados mediante densitometría ósea muestran mantenimiento o aumento de la masa ósea. Estudios que se han realizado sobre inactividad total, con sujetos sanos permaneciendo en cama durante periodos de $4-32$ horas semanales, demostraron pérdidas del $1 \%$ de la masa ósea por semana. Cuando estos comenzaron a realizar práctica física los huesos ganaron la masa perdida. (Hernández Aguillar, Chávez Cevallo, de la Concepción Torres Marín, Torres Ramírez, \& Fleitas Díaz, 2017) El cúmulo de evidencias apoya convincentemente la teoría de que el ejercicio físico regulado y controlado, protege de las enfermedades cardiovasculares, por lo que la actividad física regular es cardioprotectora. No está demostrado que sea por acción directa, pero sí asociado a la modificación de otros factores. (Cabeza, Alvarez, Guallichico, Chávez, \& Romero, 2017)

Existen estudios que atribuyen a la práctica deportiva habitual un efecto tranquilizante o de relajación, y la capacidad para reducir la ansiedad y favorecer un mejor estado de ánimo. El deporte puede eliminar los temores y disminuir la ingestión de medicamentos e incluso hacerlos innecesarios. (Cabeza, Alvarez, Guallichico, Chávez, \& Romero, 2017) (Vaca M, y otros, 2017) En la literatura revisada se plantea que la dependencia y el deterioro físico y psíquico, más que la muerte, son el fantasma de la edad senil y su mayor preocupación, por lo que se debe lograr un estado de salud que permita conservar al máximo la expectativa de vida activa y mantener un alto nivel funcional, logrando la mayor permanencia de los ancianos en las actividades sociales. (Cantillo Bustillo J. , Rodríguez Pérez, Martínez Cantillo, \& Padilla Martínez, 2019). El Dr. Barreto Ramos obtuvo permanencia de un año $(14,7 \%)$, guardando relación con el poco tiempo transcurrido de agrupados los gerontes, resultados que no coinciden con los del presente estudio. (Barreto Ramos, Morfi Semper, Reyes Figueroa, \& Cabrera Leal, 2007) 
Se debe señalar que la población no tiene hábito de realizar ejercicios físicos de forma regular, favoreciendo el sedentarismo, estos resultados no coinciden con estudios realizados en América del Norte y Sur que refieren que las personas se preocupan por realizar sistemáticamente algún tipo de entrenamiento. (Roca Moyano, 2016)

El ejercicio mejora el estado de ánimo, disminuye la depresión y la ansiedad, eleva el vigor, la autoestima y la imagen corporal, ofrece oportunidades de distracción e interacción social, y mejorara el enfrentamiento al estrés. Artículos examinados afirman que los ancianos incorporados a diferentes modalidades de actividades grupales mejoran la salud, porque los ejercicios físicos repercuten favorablemente en la osteoporosis, hipertensión arterial, trastornos circulatorios, entre otros. (Martín Aranda, 2018) Los resultados de la presente investigación no coinciden con la Dra. Hidalgo Feria (Hidalgo Feria, 2006), en Tacajó, por el mayor beneficio en la facilidad de movimientos en su muestra. Resultados similares lo obtuvieron el Dr. Rene Barrios y colaboradores en la Ciudad de la Habana. (Barrios Duarte, Borjes Mojaiber, \& Cardoso Pérez, 2003)

\section{Conclusiones.}

- Predominó el grupo de edad de 60-74 años, y el género masculino. Prevalecieron las enfermedades Osteomioarticulares, y el tiempo de permanencia de $1-5$ años. El mayor número de gerontes no realizaba ejercicios físicos antes de su incorporación a la casa de abuelos. Entre los beneficios reportados estuvieron la mejoría en las relaciones interpersonales y la facilidad de los movimientos.

\section{Referencias bibliográficas.}

Albizu-Campos Espiñeira, J. C. (2019). Envejcimiento y bono demográfico. Reto al desarrollo. Revista Nov. Pob., 15, 30.

Barreto Ramos, Jorge Lorenzo, Morfi Samper, Rosa, Reyes Figueroa, María de los Angeles, \& Cabrera Leal, Gilsa Asunción. (2007). Diagnóstico del programa de ejercicios físicos realizados en el Círculo de abuelos "Abel Santamaría". Revista Cubana de Enfermería, 23(4) Recuperado en 13 de febrero de 2021, de http://scielo.sld.cu/scielo.php?script=sci_arttext\&pid=S0864-

03192007000400004\&lng=es\&tlng=es.

Barrios Duarte, R., Borjes Mojaiber, R., \& Cardoso Pérez, L. d. (2003). Beneficios percibidos por adultos mayores incorporados al ejercicio. Recuperado el 9 de Febrero de 2021, de Revista Cuaba de Medicina General Integral: http://scielo.sld.cu/scielo.php?script=sci_arttext\&pid=S0864-

21272003000200007\&Ing=es

Cabeza, M., Alvarez, J., Guallichico, P., Chávez, J., \& Romero, E. (2017). Entrenamiento funcional y recreación en el adulto mayor: influencia en las capacidades y habilidades físicas. Revista Cuaba de Investigaciones Biomédicas., 36(4). Obtenido de Revista Cuabana de Investigaciones Biomédicas. 
Cantillo Bustillo, J., Rodríguez Pérez, Y., Martínez Cantillo, Y. M., \& Padilla Martínez, F. (Diciembre de 2019). Evaluación funcional del adulto mayor en la comunidad. Recuperado el 9 de Febrero de 2021, de Revista Ciencias Médicas: http://scieloprueba.sld.cu/scielo.php?script=sci_arttext\&pid=S156131942019000600876\&Ing=es

Cantillo Bustillo, J., Rodríguez Pérez, Y., Martínez Cantillo, Y. M., \& Padilla Martínez, F. (1 de Marzo de 2019). Evaluación funcional del adulto mayor. Recuperado el 9 de Febrero de 2021, de Revista de Ciencias Médicas: http://revcmpinar.sld.cu/index.php/publicaciones/article/view/3950

Escalante Candeaux, L., Medina Alvarez, M., Pila Hernández, H. J., \& Gómez Valdés, A. (2019). El adulto mayor practicante sistemático: pruebas para evaluar su condición física. Recuperado el 9 de Febrero de 2021, de Revista Podium: http://scieloprueba.sld.cu/scielo.php?scrip=sci_arttext\&pid=S199624522019000300372\&Ing=es

Godoy del LLano, A., Casanova Moreno, M. d., Alvarez Lauzarique, M. E., Oliva González, Y., \& Rodríguez Hernández , N. (2018). Evaluación subprograma de atención comunitaria al adulto mayor en el muncipio Pinar del Rio. Recuperado el 9 de Febrero de 2021, de Revista de Ciencas Médicas: www.revcmpinar.sld.cu/index.php/publicaciones/article/view/3544

Hernández Aguillar, B., Chávez Cevallo, E., de la Concepción Torres Marín, J., Torres Ramírez, A., \& Fleitas Díaz, I. M. (Diciembre de 2017). Evaluación de un programa de actividad físico recreativa para el bienestar físico mental del adulto mayor. Recuperado el 9 de Febrero de 2021, de Revista Cubana de Investigaciones Biomédicas: $\quad$ http://scielo.sld.cu/scielo.php?script=sci_arttext\&pid=S086403002017000400007\&Ing=es

Hernández Ugalde, F., Alvarez Escobar, M. d., Martínez Leyva, G., Junco Sánchez, V. L., Valdés Gasmuri, I., \& Idalgo Ruiz, M. (s.f.). Polifarmacia en el anciano. Retos y soluciones.

Hidalgo Feria, Y. (2006). Funcionamiento de seis círculos de abuelos en el período 20042005. Consejo Tacajó. Tesis, Holguin.

Martín Aranda, R. (Octubre de 2018). Actividad física y calidad de vida en el adulto mayor. Una revisión narrativa. Recuperado el 9 de Febrero de 2021, de Revista Habanera de Ciencias Médicas: http://scielo.sld.cu/scielo.php?script=sci_arttext\&pid=S1729519X2018000500813\&Ing=es

Montes de Oca García, D., Bravo, A., \& Díaz Medina, C. (2008). Influencia del círculo de abuelos en la evolución de las enfermedades articulares. 23(3), 9-14. Obtenido de Revista Cubana de Enfermería.

Oficina Nacional de Estadística e Información. (2019). Anuario Estadístico de Cuba. La Habana.

Ortega Marquez, Y. (Noviembre de 2010). Influencia de la casa de abuelos sobre el adulto mayor. Recuperado el 9 de Febrero de 2021, de MEDISAN: 
http://scieloprueba.sld.cu/scielo.php?script=sci_arttext\&pid=S102930192010000800018\&Ing=es

Piña Moreno, Y., Cordero Ortero, C. D., Rodríguez Lemus, O., Izquiero Izquierdo, A., \& Vega Pérez, A. R. (Agosto de 2019). Evaluación del subprograma de atención comunitaria al adulto mayor en Mantua. Recuperado el 9 de Febrero de 2021, de Revita de Ciencias

Médicas:

http://scieloprueba.sld.cu/scielo.php?script=sci_arttext\&pid=S156131942019000400501\&Ing=es

Roca Moyano, R. E. (2016). Activiudad Física y salud en el adulto mayor de seis países latinoamericanos. Revista de Ciencias de la Actividad Física UCm, 1(17), 77-86.

Vaca M, R., Gómez R, V., Mena F, M., Yandún S, V., Realpe Z, E., \& al, e. (2017). Estudio comparativo de las capacidades físicas del adulto mayor: rango etáreo vs actividad física. Revista Cubana de Investigaciones Biomédicas., 36(1).

Zavala G, M., Vidal G, D., Catro S, M., Quiroga , P., \& Klasen P, G. (2006). Funcionamiento social del adulto mayor. Recuperado el 9 de Febrero de 2021, de Revista de Ciencias de la enfermería.: http://scielo.conisyt.cl/scielo.php?script=sci_arttext\&pid=S071795532006000200007\&Ing=es 


\section{PARA CITAR EL ARTÍCULO INDEXADO.}

Soria Pérez, R., Mestre, J. de A., Díaz Camellón, D. J., \& Santana García, Y. (2021). Ejercicio físico en adultos mayores incorporados a la Casa de Abuelos . Anatomía Digital, 4(2), 68-77. https://doi.org/10.33262/anatomiadigital.v4i2.1618

\section{Liencia}

El artículo que se publica es de exclusiva responsabilidad de los autores y no necesariamente reflejan el pensamiento de la Revista Anatomía Digital.

El artículo queda en propiedad de la revista y, por tanto, su publicación parcial y/o total en otro medio tiene que ser autorizado por el director de la Revista Anatomía Digital.
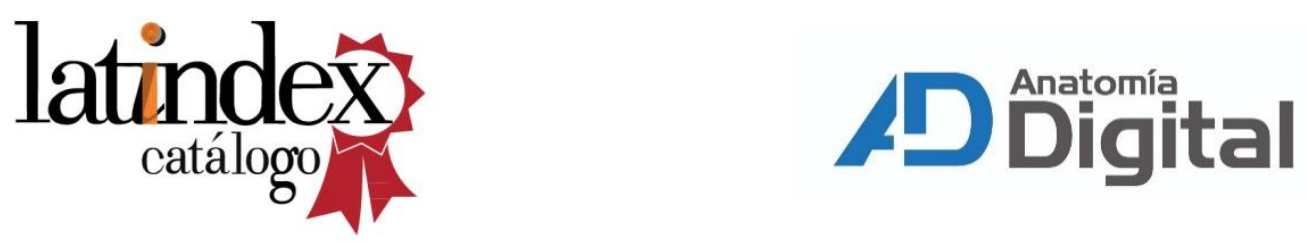